\title{
Evaluation of a Family Medicine Transitional Care Service Line
}

\author{
Bryan Farford, DO, Sally Ann Pantin, MD, John Presutti, DO, and \\ Colleen S. Ball, MS
}

Introduction: As of 2013, the all-cause readmission rate among Medicare fee-for-service beneficiaries was $\mathbf{1 7 . 5 \%}$. In addition to poor outcomes, 30-day hospital readmissions account for over $\$ 17$ billion in Medicare expenditures. The presence and involvement of a primary care provider can be essential during the transition period from hospital discharge to the outpatient setting.

Objective: In an effort to reduce 30-day hospital readmissions a transitional care management (TCM) service was implemented in a multi-site family medicine practice.

Methods: The transitional care service line was structured after the 2013 Centers for Medicare \& Medicaid Services recommended process for transitioning patients from an inpatient to an outpatient setting. The service included a care team RN, electronic documentation in an electronic medical record and the primary care physician.

Results: The 30-day readmission rate was $12.0 \%$ in the 10 months before implementation of the new service line and $12.4 \%$ in the first 10 months after implementation of the new service line. There was no evidence of an impact of the new service line on a decline in 30 -day readmission rates $(P=.18)$.

Discussion: Hospital readmissions generate unnecessary costs and often present a major burden on patients and their families. Early engagement with patients after hospital discharge will help to address any acute needs, verify medication adherence and ensure that necessary equipment and services are available.

Conclusion: Although there was no evidence of an impact of the new service line on a decline in 30day readmission rates it was decided that this service was a benefit to the patients and the physicians involved. (J Am Board Fam Med 2019;32:619-627.)

Keywords: Patient Discharge, Patient Readmission, Patient Transfer, Primary Care Physicians, Quality Improvement, Transitional Care

The Centers for Medicare \& Medicaid Services (CMS) defines a hospital readmission as an unplanned readmission for any cause to an acute care hospital within 30 days of discharge from a shortstay acute-care hospital. ${ }^{1}$ As of 2013 , the all-cause readmission rate among Medicare fee-for-service beneficiaries was $17.5 \% .^{2}$ Oftentimes hospital readmissions can be avoided and are the result of a

This article was externally peer reviewed.

Submitted 14 September 2018; revised 15 February 2019; accepted 26 February 2019.

From the Department of Family Medicine, Mayo Clinic Florida, Jacksonville, FL.

Funding: none.

Conflict of interest: none declared.

Corresponding author: Bryan Farford, DO, Department of Family Medicine, Mayo Clinic Florida, 4500 San Pablo Road, Jacksonville, FL 32224 (E-mail: Farford.Bryan@mayo.edu). disjointed care transition from the hospital to the outpatient setting. In many instances, readmissions are the result of untimely clinician followup. ${ }^{3}$

The involvement of a primary care provider (PCP) can be essential during this transition period. It has been demonstrated that lack of timely PCP followup (within 4 weeks of discharge) is associated with increased hospital readmission for the same condition and is likely to result in a longer hospitalization. ${ }^{4}$ During the postdischarge period, patients are at increased risk for adverse events related to medication errors, poor followup on pending tests at discharge, and missed opportunities for recommended testing or procedures as outpatients. ${ }^{5}$ Many of these adverse events could easily be prevented with appropriate hospital followup with their PCP. Furthermore, patients often have 
symptoms or complications as a result of their hospitalization that could be addressed with simple strategies. ${ }^{6}$

In addition to poor outcomes, 30-day hospital readmissions account for over $\$ 17$ billion in Medicare expenditures. ${ }^{7}$ As of October 1, 2012, in response to these findings, the Affordable Care Act established the Hospital Readmissions Reduction Program, which required CMS to reduce payments to hospitals with excessive readmissions. ${ }^{8}$ Moreover, in January 2013 CMS began reimbursing health care providers for care management services provided to patients following a discharge from an inpatient hospital setting. ${ }^{9}$ Two Current Procedural Terminology (CPT) codes (99495 and 99496) were established to report the transitional care management services provided following a hospital discharge. ${ }^{10}$ To use the CPT codes, 3 major requirements must be met. First, interactive contact must be made with the patient and/or caregiver, as appropriate, within 2 business days following the patient's discharge from the inpatient setting. The contact can be made by any competent clinical staff and can occur via telephone, e-mail, or a face-to-face visit. The second requirement is to provide nonface-to-face services to the patient, unless it is determined that they are not medically indicated or needed. A clinician or clinical staff with supervision may do this. The third element is to provide 1 face-to-face visit within certain timeframes as described by the CPT codes. A face-toface visit within 14 days of discharge with moderate medical decision complexity is CPT 99495. A faceto-face visit within 7 days of discharge with high medical decision complexity is CPT $99496^{10}$ (Table 1).

\section{Objective}

The primary objective of this quality improvement project was to determine if implementation of a transitional care management service that aligned with the guidelines recommended by CMS would decrease 30-day hospital readmissions when compared with the previous model for hospital follow-up care.

\section{Methods}

\section{Practice Profile}

The department of Family Medicine at Mayo Clinic Florida consists of a residency training location and 3 satellite community-based practices.
This residency training program includes 10 staff physicians, 18 residents and 2 advanced-practice providers. The community-based practices consist of 3 separate clinics that are located geographically within 45 minutes of one another with a total of 15 physicians and 8 advanced-practice providers. In sum, the Department has a panel of 34,075 patients between newborn age and 103 years of age. Of these patients, $57 \%$ are women, and $6 \%$ are less than 18 years old.

Each location admits patients to the Mayo Clinic Florida hospital who are managed by the Family Medicine Service. This service consists of family medicine residents and attending family medicine staff physicians. Historically, patients that were discharged from the family medicine hospital service had follow-up visits ordered by the residents discharging the patients. The respective office would then contact the patients and arrange a date and time for followup with their PCP. No emphasis was placed on the timeliness of the follow-up visit unless the discharging team had specific concerns. No other contact was made with the patients until their office visit with their PCP.

If patients were discharged from a subspecialty service other than the Family Medicine service, followup was unlikely to happen with the PCP unless the patient requested followup. However, most patients would have an outpatient followup arranged with the discharging service.

\section{Transitional Care Management (TCM) Implementation}

In August 2016 a new process was implemented to better align with the CMS requirements for transitional care management services. The first step in the new process was to assign a care team $\mathrm{RN}$ to the transitional care management (TCM) service line at each location. The care team $\mathrm{RN}$ would be responsible for contacting patients that were discharged from the hospital within 2 business days of the discharge. Contact could be made either by a phone call or, if the patient was enrolled in the Patient On-line Services portal, then an on-line portal message could be utilized. To document the communication and to ensure the review of pertinent elements of the hospital discharge, an electronic form was created that would be saved to the electronic medical record (EMR) under a specific note type that was easily accessible for the PCP. 
Table 1. Necessary Requirements to Bill for Transitional Care Services Using 99495 and 99496

Step 1

Interactive Contact with the Patient or Caregiver

Within 2 business days following discharge from an inpatient setting to an outpatient setting.

Contact may be way of a telephone call, email or face-to-face visit.

Can be accomplished by the provider or competent clinical staff.

- Attempts to make contact should continue after the first 2 business days if initial contact is unsuccessful.

If two or more separate contact attempts are made unsuccessfully and documented in the medical record one may bill for the TCM service if all other elements are met.

Step 2

Non-Face-to-Face Services Provided by Physicians or APPs

Acquire and review discharge information (e.g., discharge summary or continuity of care documents).

Review need for or follow-up on pending diagnostic tests and treatments.

- Communicate with other health care professionals who will assume or reassume care of the patient's system-specific problems.

Provide education to the patient, family, guardian, and/or caregiver.

Establish or re-establish referrals and arrange for needed community resources.

Assist in scheduling required follow-up with community providers and services.

Non-Face-to-Face Services Provided by Clinical Staff Under the Direction of a Physician or APP

- Communication with agencies and community services the patient uses.

- Provide education to the patient, family, guardian, and/or caretaker to support self-management, independent living, and activities of daily living.

Evaluate and support treatment regimen adherence and medication management.

Identify available community and health resources.

Assist the patient and/or family in accessing needed care and services.

Step 3

Face-to-Face Visit

A face-to face visit is a required element of TCM billing.

- Services with moderate medical decision complexity with a face-to-face visit within 14 days of discharge use CPT code 99495.

- Services with high medical decision complexity with a face-to-face visit within 7 days of discharge use CPT code 99496.

- For eligible telehealth services, the use of a telecommunications system substitutes for an in-person encounter.

APP, Advanced Practice Provider; CPT, Current Procedural Terminology; TCM, transitional care management.

The key components of the communication included medication review and reconciliation, review of necessary equipment or services, and assurance that the patient had a follow-up visit with their PCP within 14 days of discharge (Figure 1). If the care team $\mathrm{RN}$ uncovered any issue that required PCP involvement before the follow-up visit they would send a message to the PCP.

To ensure efficiency, it was determined that only patients with a moderate-to-high risk of hospital readmission would be enrolled in the TCM service line. A calculated readmission score assigned to the patient during their hospitalization determined risk of readmission. The LACE index scoring tool was used to determine the risk of readmission. This scoring system uses length of stay, acuity of admission, comorbidities, and emergency department visits (Figure 2). Furthermore, a patient discharge pool was developed within the EMR that electronically collects patients discharged from the Mayo Clinic hospital with PCPs in the department of Family Medicine and a moderate to high risk for readmission. The care team $\mathrm{RNs}$ were then instructed to monitor the pool daily for patients assigned to their practice site being discharged from the hospital.

The final step in the implementation process was to have these patients receive a face-to-face followup with their assigned PCP within 14 days of discharge. If the care team $\mathrm{RN}$ determined the patient needed close followup or the patient was at high risk for readmission, a follow-up visit was scheduled within 7 days of discharge. In the rare instance that the PCP was not available for followup the patient would see another physician in the practice. 
Figure 1. Electronic form used by care team RN to document discharge follow-up communication.

Result Type: Hosp Nursing Care Mgmt/Care Coord

Result Date: 11 January 2017 14:04 EST

Result Status: $\quad$ Auth (Verified)

Result Title: Discharge Follow Up - Risk for Readmission

Performed By:

*Final*

Discharge Follow Up - Risk for Readmission Entered On: 12-Jan-2017 14:12 EST

Performed On: 11-Jan-2017 14:04 EST by

Phone Call

Follow up attempted: First attempt

Follow up completed: Via telephone

Reason For Hospitalization : Recurrent tachycardia

Call placed to : patient

Telephone Number:

Can patient name medical condition : Yes AND has clear understanding

Admission/Discharge Dates : 01/10/17 19:43 01/11/17 19:52

Have admit symptoms improved?: Yes

How does patient feel they are managing? : 3 . No questions/concerns

Medications

Patient able to fill take home meds : Yes

Medication List

(As Of: 12-Jan-2017 14:12:13 EST)

Normal Order

ACETAMINOPHEN TAB, $325 \mathrm{mg}$

ACETAMINOPHEN TAB, 325 mg ; Status: Discontinued ; Ordered As Mnemonic: Tyleno Simple Display Line: 650 mg, 2 Tab, PO, Q4H, PRN: Headache

Medication list reviewed? : Yes

Patient taking meds as prescribed?: Yes

Controlled Substances: No

Antibiotics: No

Diabetic Oral Meds : No

Is the patient taking Insulin: No

Is patient taking Low Molecular Weight Heparin : No

Is the patient taking Warfarin: No

\section{Anticoag Meds}

Is the patient taking anticoag meds as instructed?: Yes

Recent signs/symptoms of bleeding or clotting: No

Low Molecular Weight Heparin Comment: Xarelto

Previous INR Result : INR (02/09/2015 08:25): 1.1

Who assists patient with meds : Self-managed

Does pt request additional med assistance?: No

Does pt/caregiver have med questions? : No

\section{Equipment/Services}

Does patient have ambulatory difficulties?: No

Does patient have durable med equipment?: None

Was new med equipment ordered at discharge?: No

Has patient obtained med equipment at discharge?: N/A

Patient using following services: None

Follow-up

Primary Care Provider Appointment : 17-Jan-2017 14:30 EST

Primary Care Follow Up Provider:

Primary Care Provider Follow up appt location: Beaches Primary Care

Is there reason patient can't keep appointment?: No

Does the patient understand the DC instructions?: Yes

Discharge instructions reinforced with patient?: Yes

Discharge summary concerns/advice summarize: Pt states she is feeling much better after her 10th cardioversion. She is taking all

medications as prescribed, aware of medication changes, confirmed PCP f/u appt. Will call if symptoms return.

Patient given number to call for questions : Yes

\section{Data Collection}

Data regarding hospital 30-day readmission rates were collected through the Practice Management Tool (PMT), a central hub used to collect and analyze data on primary care patients. The PMT was developed by Enterprise Analytics (Rochester, $\mathrm{MN}$ ) and allows Primary Care health care administrative and clinical staff access to data 
Figure 2. LACE Index Scoring Tool.

Step 1. Length of Stay

Length of stay (including day of admission and discharge): days

\begin{tabular}{|c|c|}
\hline Length of stay (days) & Score (circle as appropriate) \\
\hline 1 & 1 \\
\hline 2 & 2 \\
\hline 3 & 3 \\
\hline $4-6$ & 4 \\
\hline $7-13$ & 5 \\
\hline 14 or more & 7 \\
\hline
\end{tabular}
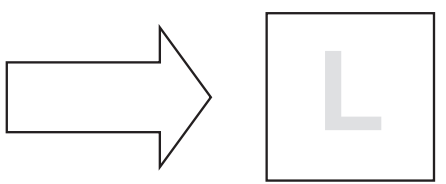

Step 2. Acuity of Admission

Was the patient admitted to hospital via the emergency department?

If yes, enter " 3 " in Box $A$, otherwise enter " 0 " in Box A

Step 3. Comorbidities

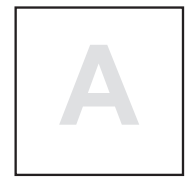

\begin{tabular}{|c|c|c|}
\hline Condition (definitions and notes on reverse) & Score (circle as appropriate) & \multirow{16}{*}{$\begin{array}{c}\text { If the TOTAL score is between } 0 \text { and } \\
3 \text { enter the score into Box C. If the } \\
\text { score is } 4 \text { or higher, enter } 5 \text { into Box } \\
\text { C }\end{array}$} \\
\hline Previous myocardial infarction & +1 & \\
\hline Cerebrovascular disease & +1 & \\
\hline Peripheral vascular disease & +1 & \\
\hline Diabetes without complications & +1 & \\
\hline Congestive heart failure & +2 & \\
\hline Diabetes with end organ damage & +2 & \\
\hline Chronic pulmonary disease & +2 & \\
\hline Mild liver or renal disease & +2 & \\
\hline Any tumor (including lymphoma or leukemia) & +2 & \\
\hline Dementia & +3 & \\
\hline Connective tissue disease & +3 & \\
\hline AIDS & +4 & \\
\hline Moderate or severe liver or renal disease & +4 & \\
\hline Metastatic solid tumor & +6 & \\
\hline TOTAL & & \\
\hline
\end{tabular}

Step 4. Emergency department visits

How many times has the patient visited an emergency department in the six months prior to admission (not including the emergency department visit immediately preceding the current admission)?

Enter this number or 4 (whichever is smaller) in Box $\mathrm{E}$

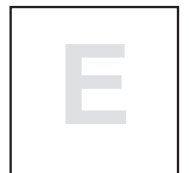

Add numbers in Box L, Box A, Box C, Box E to generate LACE score and enter into box below. If the patient has a LACE score is greater than or equal to 10 the patient can be referred to the virtual ward

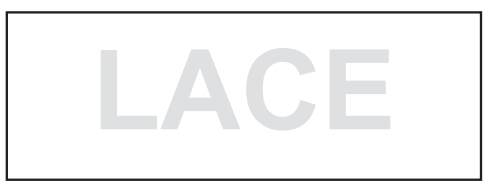

LACE Score Risk of Readmission: 0 - 4 Low, 5 - 9 Moderate, > 9 High Risk 
necessary to align and achieve goals brought forth by the practice. Data analysis for the 30-day hospital readmission began in October 2015 and were reported on a monthly basis thereafter through June 2017.

In addition, the Tableau Report workbook was used to review hospital readmission details including admitting diagnosis.

\section{Statistical Analysis}

To evaluate the impact of the TCM service line on 30-day readmission rates, an interrupted time series analysis was utilized using a segmented regression approach. The model used was:

Rate $_{\mathrm{t}}=\beta 0+\beta 1 \times$ time $_{\mathrm{t}}+\beta 2 \times$ intervention $_{\mathrm{t}}+$ $\beta 3 \times$ time after intervention ${ }_{\mathrm{t}}+\varepsilon_{\mathrm{t}}$

where time is the number of months from the start of the study to time $t$, intervention is 0 before implementation of the TCM service line (August 2016) and one otherwise, and time after the intervention is the number of months after implementation of the TCM service line and 0 if before August 2016. Data were available from January 2015 through June 2017; however, data before October 2015 were excluded to have the same number of time points before and after implementation of the TCM service line.

\section{Results}

During the period evaluated before implementation of the TCM service (October 2015 to July 2016) there were 1702 Family Medicine patients discharged from the hospital. During this 10-month period the 30 -day readmission rate was $12.0 \%$. Following implementation of the TCM service (August 2016 to June 2017) there were a total of 2076 Family Medicine patients discharged from the hospital service and the 30 -day readmission rate was $12.4 \%$. There was no evidence of an impact of the new service line on a decline in 30-day readmission rates $(P=.18)$ (Table 2, Figure 3).

In addition, during the first and second quarter of 2017, a comparison was made between the readmission rates of those meeting criteria for enrollment in TCM (patients with a moderate to high risk of hospital readmission) and those who did not. The readmission rate during this time for patients not meeting criteria was only $5.5 \%$ versus $13 \%$ for those meeting criteria.
Table 2. Segmented Regression Looking at Impact of New Service Line on 30-Day Readmission Rates

\begin{tabular}{lrcc}
\hline Variable & Coefficient & SE & $P$ Value \\
\hline Time & -0.00407 & 0.00200 & .057 \\
Intervention & 0.02323 & 0.01630 & .17 \\
Time after intervention & 0.00369 & 0.00262 & .18 \\
\hline
\end{tabular}

SE, standard error

The number 1 admitting diagnosis both before and after implementation of the TCM service was septicemia. The second most common admitting diagnosis was diverticular disease/intestinal obstruction before the TCM service implementation, and congestive heart failure post implementation. The 2 most common diagnoses on 30-day readmission before TCM were gastrointestinal-related illness and pneumonia. Following deployment of the TCM service the 2 most common diagnoses for 30-day readmission were congestive heart failure and acute renal failure.

The care team $\mathrm{RN}$ was able to make contact with $69 \%$ of the patients enrolled in TCM. The study did not differentiate whether or not patients were contacted by phone or through the on-line portal. Fifty-two percent of the patients enrolled in the TCM service line completed their follow-up visit with their PCP within 14 days of discharge.

\section{Limitations}

There were 4 limitations identified in the effectiveness of implementing a TCM service. The first was that only patients admitted to the Mayo Clinic Hospital in Florida were enrolled into TCM. Currently, there is not an effective way of identifying patients that are admitted or discharged from other hospital systems. In most instances the patient notified the PCP once they were discharged from another inpatient facility. Very seldom is their communication from the external hospital when a patient is admitted or discharged. As a result it was not possible to enroll these patients effectively into the TCM service.

Second, some patients were admitted to the Mayo Clinic Hospital under another service (eg, orthopedic surgery) and had follow-up with the discharging service. Despite having a PCP in the department of Family Medicine it was determined that these patients would be excluded from TCM. 
Figure 3. Fitted lines of the segmented regression analysis of interrupted time series for the monthly 30-day readmission rates, October 2015-June 2017. Open circles represent the observed 30-day readmission rates. The solid line represents the fitted 30-day readmission rate before implementation of the transitional care management service. The dashed line represents the fitted 30-day readmission rate after implementations of the transitional care management service.

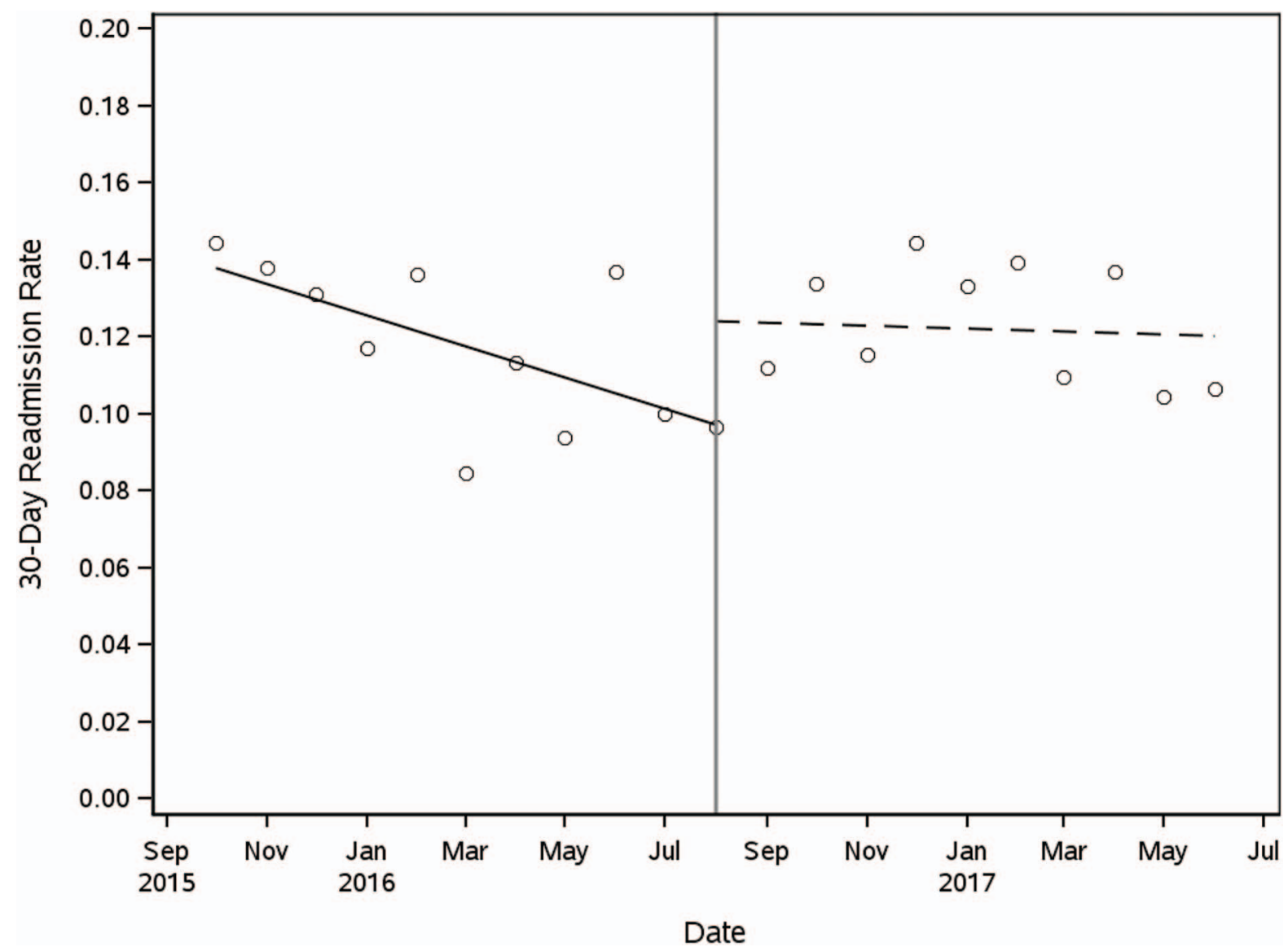

The third limitation was that some patients being discharged from the hospital were sent to step down care facilities such as skilled nursing and inpatient rehabilitation facilities. Although these patients were not excluded from the TCM service and the care team $\mathrm{RN}$ did attempt to contact the patients; follow up within 14 days was often difficult to arrange. In some cases patients were sent back to the hospital for readmission from these facilities before the PCP being able to see them for a follow-up visit.

Finally, the care team RN only contacted patients Monday through Friday. If a patient was discharged over the weekend they were not contacted until the following workweek. Although, this did not occur often it could be a potential limitation in the effectiveness of this TCM model.

\section{Discussion}

Hospital readmissions often present a major burden on patients and their families. In addition, these readmissions frequently generate unnecessary costs and have a negative financial impact on the health care system. In an attempt to improve the 30-day readmission rate for the department of Family Medicine at Mayo Clinic Florida a TCM service was initiated based on CMS guidelines.

Before implementing the TCM service line, the rate of readmission was $12.0 \%$, and following implementation the rate of readmission was $12.4 \%$. Although there was no evidence of an impact of the new service line on a decline in 30-day readmission rates it was decided that this service was a benefit to the patients and the physicians involved. Having the care team $\mathrm{RN}$ contact the patients shortly after 
discharge, although it may not have prevented readmission, ensured patients were taking their medications appropriately, had the necessary equipment and supplies following discharge and were receiving any necessary services such as home health care. When necessary, the care team $\mathrm{RN}$ provided valuable clinical feedback to the PCPs, addressed patients' questions and concerns, and triaged patients to appropriate follow-up visits.

In addition, the rate of readmission, $12.4 \%$, was well below the national average of $17.5 \%$ and this represents a good model for practices struggling with high 30-day readmission rates. In this model no additional staffing was required and the practices did not accrue any additional costs. Physicians were also educated on billing visits using the CPT codes 99495 and 99496 when appropriate. Although a cost analysis was not performed it was anticipated that there was additional revenue generated for the services rendered.

It was determined that enrolling patients into TCM was most beneficial if they were at moderateto-high risk of hospital readmission. Only $5.5 \%$ of the patients not meeting these criteria were readmitted during the first 2 quarters of 2017. It was decided that the value gained was nominal compared with the amount of resources that it would consume had these patients also been enrolled in TCM.

Other institutions have been successful with similar TCM models. Four primary care practices in Oregon were able to reduce their 30-day readmission rate from $27 \%$ to $7.1 \%$ by using an $\mathrm{RN}$ (care manager) to manage patients being discharged from the hospital. The care managers were responsible for managing communication from the inpatient team, creating a hospital follow-up workflow, developing questions for patients being discharged and calling patients within 2 to 3 days to ensure a follow-up appointment was scheduled within 7 days of discharge. Readmission risk was not considered in this design. ${ }^{11}$ Having all patients follow up within 7 days of discharge may have made an impact on the readmission rate and should be considered further when attempting to implement a new TCM model.

In North Carolina, a large academic family medicine practice utilized a clinical pharmacist in their workflow to contact patients following discharge to perform medication reconciliation and address medication related questions. In addition to the pharmacist, a nurse contacted the patient by phone within 2 business days of discharge to ensure adequate physician follow-up and to address any patient related health concerns. Follow-up was based on readmission risk where moderate risk patients followed up within 14 days and high-risk patients were seen at follow-up within 7 days. The implementation of this TCM model reduced hospital readmission rates from $14.2 \%$ to $5.3 \% .^{12}$ In this model the utilization of a clinical pharmacist appeared to make a significant impact on the rate of readmission. However, the cost effectiveness of adding a pharmacist or other components (eg, social workers, health coaches) to the model should be considered before implementation.

\section{Conclusion}

To improve the quality of care and to decrease national health care costs, it is imperative that hospital readmission rates remain low. Having early engagement with patients after they are discharged from the hospital will help to address any acute needs, verify medication adherence and ensure that necessary equipment and services are available. In addition, close follow up with a PCP following discharge will increase the likelihood that required labs, tests and specialty consults are completed and addressed. Currently, follow up within 14 days seems to be a reasonable time frame however closer follow up may yield lower readmission rates especially in higher-risk patients.

The implementation of a TCM model requires few resources however; communication between the discharging facility and the outpatient primary care practice is often fragmented. Having a shared EMR system improves this communication and was a key element of this TCM service. Regardless of the EMR, electronic communication should be a standard of care initiative to make certain that patients discharged from hospitals outside of their assigned system get adequate followup with their PCP. This should include a detailed discharge summary including discharging diagnosis, medications at time of discharge, equipment or services that are needed after discharge, as well as any other information that is pertinent to the discharge.

Furthermore, for patients being discharged to a step-down facility, it is important to have early engagement with the patients, caregivers and health care workers of the facility. Followup with a 
PCP as quickly as possible should remain a priority for the health care team. Having early contact and communication may reduce unnecessary emergency department visits and readmissions.

This communication with the addition of a dedicated $\mathrm{RN}$ to contact patients following discharge and adequate outpatient follow up with the patient's PCP should reduce the national rate of 30day hospital readmissions. Lower readmission rates may be achieved by adding a clinical pharmacist to the TCM workflow but further evaluation, including cost, should take place before making this argument.

To see this article online, please go to: http://jabfm.org/content/ 32/4/619.full.

\section{References}

1. CMS.gov. 2015 measure information about the 30day all-cause hospital readmission measure, calculated for the value-based payment modifier program 2015. Available from: https://www.cms.gov/Medicare/Medicare-Fee-for-Service-Payment/PhysicianFeedbackProgram/Downloads/2015-ACR-MIF.pdf. Accessed May 27, 2018.

2. Innovation.CMS.gov. 2014. Available from: https:// innovation.cms.gov/Files/reports/patient-safetyresults.pdf. Accessed May 27, 2018.

3. RWJF.org. The revolving door: A report on U.S. hospital readmissions. 2013. Available from: https:// www.rwjf.org/content/dam/farm/reports/reports/ 2013/rwjf404178. Accessed May 27, 2018.

4. Misky GJ, Wald HL, Coleman EA. Post-hospitalization transitions: Examining the effects of timing of primary care provider follow-up. J Hosp Med 2010;5:392-7.
5. Moore C, Wisnivesky J, Williams S, McGinn T. Medical errors related to discontinuity of care from an inpatient to an outpatient setting. J Gen Intern Med 2003;18:646-51.

6. Forster AJ, Murff HJ, Peterson JF, et al. The incidence and severity of adverse events affecting patients after discharge from the hospital. Ann Intern Med 2003;138:161.

7. Zuckerman RB, Sheingold SH, Orav EJ, et al. Readmissions, observation, and the hospital readmissions reduction program. N Engl J Med 2016;374: 1543-51.

8. CMS.gov. Readmissions Reduction Program (HRRP)_Centers for Medicare \& Medicaid Services. 2018. Available from: https://www.cms.gov/ medicare/medicare-fee-for-service-payment/acute inpatientpps/readmissions-reduction-program. html. Accessed May 27, 2018.

9. CMS.gov. Frequently asked questions about billing the Medicare physician fee schedule fortransitional care management services. March 17, 2016. Available from: https://www.cms.gov/Medicare/ Medicare-Fee-for-Service-Payment/Physician FeeSched/Downloads/FAQ-TCMS.pdf. Accessed May 27, 2018.

10. CMS.gov. Transitional care management services. December 2016. Available from: https://www.cms. gov/Outreach-and-Education/Medicare-LearningNetwork-MLN/MLNProducts/Downloads/Transi tional-Care-Management-Services-Text-Only.pdf. Accessed May 27, 2018.

11. White B, Carney PA, Flynn J, Marino M, Fields S. Reducing hospital readmissions through primary care practice transformation. J Fam Pract 2014;63: 67-73.

12. Hitch B, Parlier AB, Reed L, et al. Evaluation of a team-based, transition-of-care management service on 30-day readmission rates. N C Med J 2016;77: 87-92. 